\title{
A Low Cost Omnidirectional Relative Localization Sensor for Swarm Applications
}

\author{
Anton Kohlbacher, Jens Eliasson \\ Computer Science, Electrical and Space Engineering \\ Luleå University of Technology \\ Luleå, Sweden
}

\author{
Kevin Acres, Hoam Chung, Jan Carlo Barca \\ Monash Swarm Robotics Laboratory \\ Monash University \\ Melbourne, Australia
}

\begin{abstract}
By enabling coordinated task execution and movement, robotic swarms can achieve efficient exploration of unknown environments. In this paper, we propose a relative localization sensor system using Ultra-wideband (UWB) radio technology for ranging. This system is light-weight and relatively indifferent to the types of surrounding environments. Infrastructure dependency such as the requirement of beacons at known locations is eliminated by making an array of sensors on a swarm agent. In this paper, a novel algorithm is implemented on hardware with limited resources and compared to a more traditional trilateration approach. Both utilize Particle Swarm Optimization (PSO) to be more robust against noise and to achieve similar accuracy. The experimental results show that the proposed algorithm runs up to ten times faster than the existing trilateration approach. The sensor array which forms the localization system weighs only $56 \mathrm{~g}$, and achieves around $0.5 \mathrm{~m}$ RMSE with a $10 \mathrm{~Hz}$ update rate. Experiments show that the accuracy can be further improved if the rotational bias observed in the UWB devices are compensated for.
\end{abstract}

Index Terms-Relative Localization, UWB, PSO, Swarm Robotics, Trilateration, Angulation.

\section{INTRODUCTION}

Finding the location of objects relative to each other are important in many fields where absolute positioning cannot be achieved; such as indoors, beneath trees or otherwise GPSdenied environments. One huge application domain for relative localization is Swarm Robotics (SR), where it is a key problem to be solved to enable coordination of movement and task execution [1]. Benefits of SR are many, for example a swarm will make exploration of large remote areas faster, efficient and more reliable than a traditional approach [2]. Similar benefits also apply to e.g. disaster site management and surveillance.

There are several distinct ways to solve the localization problem, however considering the application of SR imposes some limitations. In [3] Stirling et al. observed that "Global Information" (GI) cannot be used in such a localization system, which are environmental maps, centralized processing, absolute positioning and long-range communications. Furthermore, the ambient environment might impose other restrictions such as light conditions and obstacles [4] or temperature variations [5] depending on the system.

The problem of relative localization can be divided into two distinct steps, as described by Gezici and Poor [6]. First one estimates position related parameters via some kind of sensor, and secondly one uses those parameters to estimate the position itself. How this can be achieved for a robotic swarm without GI are investigated, developed and experimentally verified with a prototype.

The remainder of this paper is arranged as follows: Section II describes related work. Section III explains how the software and hardware of our prototype localization sensor is built up. Section IV details the experimental design and performance both in simulation and a number of experiments. Lastly, Section V draws a concluding statement and suggest future improvement.

\section{RELATED WORK}

\section{A. Parameter estimation}

There are three main methods to extract positional related parameters. One is via optical sensors, the second via acoustic measurements and the third via radio frequency (RF) sensors.

Optical measurements are common in the visual or infra-red domain, and require distinguished or easily identifiable features to allow for robust operation [7]. By carrying a known 2D pattern with a diameter of $18 \mathrm{~cm}$ [4] demonstrated an accuracy as high as $1.2 \mathrm{~cm}$ and an update rate up to $60 \mathrm{~Hz}$. This required sufficient light conditions and that the pattern and sensor stayed on roughly the same altitude, with a maximum inter-distance of just $0.5 \mathrm{~m}$. Relaxing the accuracy to $6.5 \mathrm{~cm}$ and reducing update rate to $27 \mathrm{~Hz}$ the inter-distance between the nodes could be brought up to $5.5 \mathrm{~m}$ maximum.

Additionally, as the pattern is $2 \mathrm{D}$ it can only be viewed from certain angles and distances, which put strict limitations on the relative position between the two nodes. Similar restrictions are discovered in [3] where infrared lasers are used, which only performs well in dark or shaded conditions and in a clutter-free environment.

The second and third method for parameter extraction is quite similar, where different kind of transducers are used to measure range related information such as propagation time. Acoustic measurements have some disadvantages in that it needs larger nodes and higher computational power for the same type of accuracy as RF [8],[9]. Although it should be noted that it relaxes the resolution required for extracting time related information, as acoustic waves travel far slower than radio waves, and thus affects the distance information less. Another way to measure range related information is to make use of received signal strength (RSS), but it has a much lower accuracy 
than time-based approaches and is therefore less relevant [10]. While allowing for more flexibility, RF ranging has some drawbacks in that it could be bandwidth-limited due to safety rules and interference mitigation, and suffer from multipath environments or non-line-of-sight (NLoS) errors [4]. This might cause the direct path (DP) to be impaired by way of attenuation, reflections of obstacles or occlusions, which affects the range estimation. Synchronization and clock drift also plays an important role as each nanosecond corresponds to roughly a $30 \mathrm{~cm}$ distance due to propagation with speed of light.

To address these various errors, Ultra-wideband (UWB) radios have been identified as a novel standard for precision ranging [11]. It is included in the IEEE 802.15.4a standard, where companies have developed it to perform range measurements with a $10 \mathrm{~cm}$ precision in line of sight (LoS) conditions for distances up to hundredths of meters [12].

\section{B. Positional estimation}

To estimate the relative position, one commonly makes use of range or angle estimates from a distant node with respect to a set of reference nodes with known positions, referred to as anchors. To then acquire a distant source position from range measurements the following equation can be used in a trilateration scheme, where $d_{i}$ are the i-th distance between the source position $s=\left[x_{s}, y_{s}, z_{s}\right]$ and the known anchor position $p_{i}=\left[x_{i}, y_{i}, z_{i}\right]$

$$
d_{i}=\left(\left(x_{i}-x_{s}\right)^{2}+\left(y_{i}-y_{s}\right)^{2}+\left(z_{i}-z_{S}\right)^{2}\right)^{1 / 2}=\left\|p_{i}-S\right\| .
$$

Solving this equation with a minimum of four anchors, to account for the flip ambiguity, the source position can be found. Although due to the sources of error a noise component is added to $d_{i}$ so that $m_{i}$ will be the actual measured distance. By modelling the function as a cost function for minimization

$$
s_{\text {est }}=\arg \min _{s} \sum\left[\left\|p_{i}-s\right\|-m_{i}\right]^{2}
$$

the positional ambiguity caused by the noise can then be minimized to find estimated position $s_{\text {est }}[13]$. To find this minimum there are two different methods; deterministic and stochastic. The deterministic approach usually starts with an initial guess that then, at each iterative step, moves closer to the optimal solution by evaluating the objective function. This form of gradient descent search to minimize the cost function has a problem of getting trapped in a local minimum which could pose a problem for the localization cost function, where several local minima can exist [14]. Stochastic methods can take the form of population based metaheuristics, where particles populate a search space and attempt to minimize the cost function each iteration. [15] states that even though stochastic methods have a good chance of determining the global best solution, they have some disadvantages. Automatic tuning of algorithm-specific parameters can cause inconvenience in deployment of mobile robots as there is no systematic approach. Furthermore the relatively high complexity of such kind of algorithms can make them ill-suited for real-time applications. However [16] validated that particle swarm optimization (PSO) could be used for slower real time applications and argued that it should be used if robustness were required. PSO has also been validated in several other experiments for localization, where it is found to be a good candidate for ill-conditioned problems [17],[18]. By localizing in real-time using PSO an update rate slightly above $1 \mathrm{~Hz}$ have been achieved previously by Qin et al. [17], which is significantly improved in this paper. More traditional stochastic approaches are e.g. extended Kalman filters (EKF), which are commonly used. [19] found it to have an accuracy on the same order of magnitude as particle-based algorithms, but argued that it was less robust in case of numerical instability. In [20] EKF were shown to perform well for lower amounts of noise, and positioning in nonlinear and noisy environments could be further improved by utilizing a hybrid PSO-EKF approach. In this paper PSO is used to increase robustness of the positional estimation, but a hybrid approach using EKF could also be feasible.

To reduce the computational load, a novel technique dubbed TARDIS could be used for estimating directions in 3D space [21]. Briefly explained the method makes use of an antenna array formed as a 3-simplex to find relative angles to a remote source, as depicted in Fig. 1. It uses time difference of arrival (TDOA) measurements, and a few fast computations will then determine bearing and elevation information, by utilizing the fact that the array has a circumscribed sphere of radius $R$. However, it is not robust to noise, and for swarming applications it would be useful to know not only the direction but also the position of the remote source. By making use of time of flight (TOF) measurements and then inferring TDOA from that both direction and range can be obtained, and then converted from spherical to cartesian coordinates if required. For handling noise, a proposition is to state the problem as a cost function for minimization as

$$
\begin{gathered}
\arg \min _{\theta, \varphi} \mathrm{f}(\theta, \varphi)= \\
\arg \min _{\theta, \varphi} \sum\left[x_{i} \cos (\theta) \sin (\varphi)+y_{i} \cos (\theta) \cos (\varphi)+z_{i} \sin (\theta)-c \tau_{\mathrm{i}}\right]^{2}
\end{gathered}
$$

The angle $\theta$ is elevation, which is measured from a plane parallel to the base of the tetrahedron centered at the sphere origin. Bearing, $\varphi$, is measured clockwise on the plane starting at vertex $\mathrm{A}$, and anchor positions range from $i=1, \ldots, 4$. Parameter $\tau_{\mathrm{i}}$ is the measured TDOA between the vertices, which have undergone the normalization procedure described in [21]. Lastly, $c$ is the speed of light.

There is still one major issue for relative localization in the application of SR to discuss, which is robot-to-robot localization. In many cases source localization is dependent on

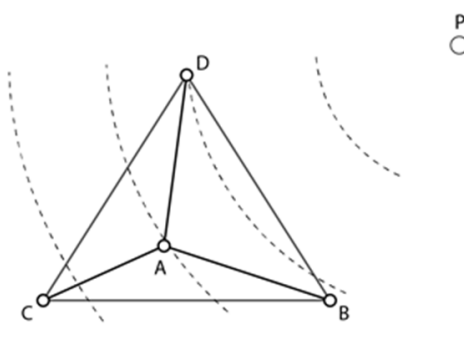

Figure 1. Tetrahedral antenna array propsed in [21]. The wavefront of the signal orginating at point $P$ transitions the vertices at varying times depending on the relative orientation of $\mathrm{P}$ with respect to the array. 
an already existing infrastructure of anchors [22], [17] and central computing [4], [8], which would make the system unscalable and reliant on GI. This puts severe limitations on a swarm, which [3] tries to avoid by alternating swarm agents between temporary anchor or moving entity. It would be still better for agents to find positional data between each other independently of their roles to not be semi-static. This could be feasible if each swarm agent was equipped with a tetrahedral antenna array, making the localization fully distributed. To share information between swarm agents, which could be viewed as a wireless sensor network (WSN), [23] have made use of a Mulle sensor node from Eistec AB. It is a deeply constrained device which makes the WSN able to communicate without using a central gateway. There is however one big drawback of making each agent carry a positioning sensor, which is that it must be able to fit onboard without being obtrusive. Thus, the size of the antenna array need to be quite small. As stated in [11] and shown with the Cramér Rao Lower Bound (CRLB), there is an accuracy limit in positional estimation constrained by sensor size and the amount of unbiased noise present. Thus there is a tradeoff between positional accuracy, scalability and computational efficiency which will be evaluated in this paper.

\section{SYSTEM DESIGN}

The proposed technology is first evaluated in simulations and then implemented in a prototype using RF transceivers. Both trilateration and TARDIS will be compared with respect to accuracy and computational efficiency. The localization sensor is constructed as in Fig. 1 with side lengths as large as possible but still small enough to fit on a moderately sized swarm agent. It should be noted that the algorithms are not constrained to radio and indifferent to the type of transducer used.

\section{A. Software design}

The constructed algorithms are developed similarly for both trilateration and TARDIS, where the noise component is assumed to be additive and the source is assumed to be moving. By utilizing a transducer to obtain absolute ranging measurements between the anchor and the source nodes, the algorithm then processes that data to obtain position of the source node.

Both methods make use of PSO for minimizing their respective objective function, where trilateration have three unknown variables $\left[x_{s}, y_{s}, z_{s}\right]$ and TARDIS two, $[\theta, \varphi]$. TARDIS also use ranging to the four vertices to estimate the distance from origin to the source which is to be located

$$
L=0.5\left(4 R^{2}-\sum m_{i}^{2}\right)^{1 / 2},
$$

where $R$ is the radius of the circumscribed sphere of the tetrahedral array, and $L$ is the estimated length between origin and source for $i=1, \ldots, 4$.

As good descriptions of PSO already exist in literature [13], [24], it will not be elaborated on details. Simply stated it distributes $M$ particles inside a search space with set boundaries $\left[l b_{d m}, u b_{d m}\right]$ for each dimension $d m=1, \ldots, N$, where $N$ is 3 or 2 depending on the trilateration or TARDIS problem. The particles will interact with each other and fly around the search-space attempting to minimize the cost function, with a velocity considering constants $c_{1}, c_{2}$ and a linearly decreasing inertial weight $w$. The search will then stop after a maximum number of iterations or a minimum change, $f_{c}$, in global fitness value over $T$ iterations.

As our application considers localization in real time and of moving nodes, some modifications has been done which previous works lack. In our approach the boundaries of the search space are limited by considering the previous history of the source node, as

$$
\begin{aligned}
& l b_{d m}=p_{d m}-a_{d m}, \\
& u b_{d m}=p_{d m}+a_{d m},
\end{aligned}
$$

where $p_{d m}$ is the previously estimated coordinate and $a_{d m}$ is defined as

$$
a_{d m}=v_{d m} t_{e a l p}+b
$$

where $b$ being a constant margin, $v_{d m}$ the previously estimated velocity of the source and $t_{\text {elap }}$ the elapsed time between previous estimation and current. Thus the search space is linearly adjusted around the previous position, considering the velocity of the source as well as the refresh rate of the algorithm. A constant $b$ is introduced to compensate for a certain amount of error, and is determined experimentally. The number of particles, $M$, distributed uniformly in the search space, are also increased or decreased by

$$
M=C+2 \operatorname{ceil}\left(a_{d m}\right)
$$

where $C$ is a constant and $a_{d m}$ the added margin. This adjustment improves robustness to noise and computational efficiency, making it suitable for real-time applications. It should be noted that this requires a relatively accurate determination of the initial position of the source, which is achieved by averaging the first $K$ measurements.

Due to the RF channel being susceptible to additive white Gaussian noise (AWGN) an exponential low pass filter is implemented for the ranging measurements. The smoothing parameter $\alpha$ between $[0,1]$ decides how much the filter will take previous values into account. It is important not to set it too low since older ranging data might not be applicable for a moving

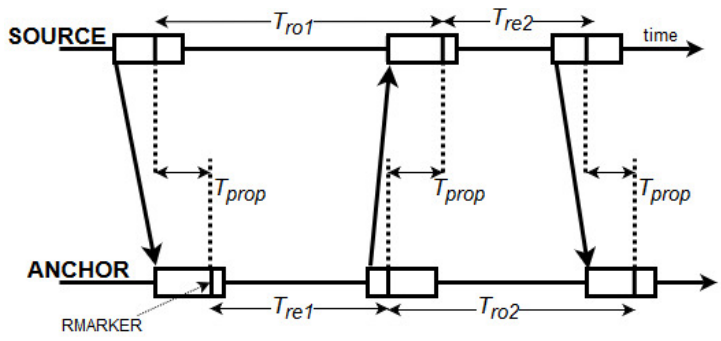

Figure 2. Two way ranging approach as implemented by [12]. 
source node. A similar filter is set for the positional output by adjusting parameter 6 . The recursive form of the filter can be seen in the equation below where $f_{i}$ is the filtered value, $k$ denotes the timestep and $m_{i}$ the noisy measurement

$$
f_{i}[k]=\alpha m_{i}[k]+(1-\alpha) f_{i}[k-1] .
$$

To obtain ranging data a series of message exchanges between the anchor and the source are used, as summarized in [12]. The general approach can be seen in Fig. 2 and is called double-sided two way ranging (DSTWR). It timestamps incoming and outgoing events with the detection of the RMARKER, and with three messages it can calculate the propagation time $T_{\text {prop }}$ via

$$
T_{p r o p}=\left(T_{r o 1} T_{r o 2}-T_{r e l} T_{r e 2}\right) /\left(T_{r o 1}+T_{r o 2}+T_{r e l}+T_{r e 1}\right) .
$$

The parameters $T_{r o}$ and $T_{r e}$ are the round trip and reply times measured by the device, which are all available to the anchor after being sent with the last message from the source. Optionally the anchor can then send a fourth message containing the calculated $T_{\text {prop}}$, which can simply be multiplied with the speed of light to obtain distance. Although seemingly a bit complex way of extracting ranging it mitigates errors due to clock drift and avoids the need of synchronization, saving a lot of deployment costs [12].

\section{B. Hardware design}

To implement the DSTWR, off-the-shelf hardware from DecaWave, called the DWM1000, is used. It is a UWB transceiver which is capable of the aforementioned accuracy of $10 \mathrm{~cm}$. This chip is connected to the Mulle, a miniature embedded internet system running Contiki, which acts as the host processor. Furthermore, the light-weight Mulle will also be used to run the positioning algorithm to avoid using any centralized processing. To communicate wirelessly between nodes and a PC running Ubuntu 16.04 LTS, the constrained application protocol (CoAP) is used.

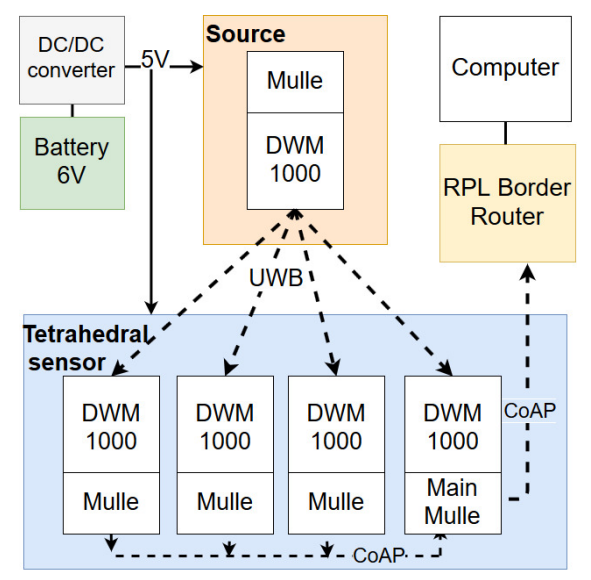

Figure 3. System overview.

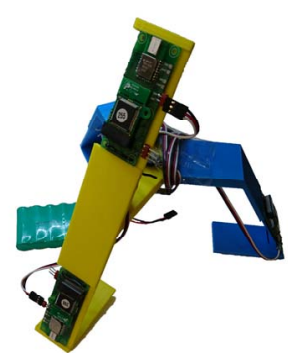

Figure 4. Tetrahedral sensor prototype, with Mulle and DWM1000 mounted at each vertex.

By using this kind of RF design, the localization sensor can be made omnidirectional and not be limited to certain view angles. Another advantage over vision-based system is robustness against ambient environment changes such as bright sunlight or dark or foggy settings. An RF based relative localization sensor also require less computations.

An overview of the system can be seen in Fig. 3, which consumes an approximate maximum of $3 \mathrm{~W}$ power during operation. A structural prototype was 3D printed to allow for accurate spacing of $0.25 \mathrm{~m}$ between each vertex, which can be seen in Fig. 4. Without the structure and cabling it had a weight of only $0.056 \mathrm{~kg}$.

\section{EXPERIMENT DESIGN AND RESULTS}

In this section both simulation and experimental results are presented. The results are evaluated with respect to the root mean square error (RMSE) which is given as

$$
R M S E=\left(\mu^{2}+\sigma^{2}\right)^{1 / 2},
$$

where $\mu$ denotes the mean error and $\sigma$ the standard deviation of the error. The vertices were chosen to be $25 \mathrm{~cm}$ apart to fit inside the rotor span of a moderately sized quadcopter. Smoothing factors were set to $\alpha=0.25, b=0.6$ and the margin $b_{d m}=0.3 \mathrm{~m}$ and $b_{d m}=0.9 \mathrm{deg}$ respectively for trilateration and TARDIS to achieve a similar type of accuracy. Figure 5 show the simulation results for these two configurations, which also includes the CRLB and the estimated position without using the PSO. The simulation was done for an arc movement of the source node with varying amounts of $\operatorname{AWGN}(0, \sigma)$ at a distance of $25 \mathrm{~m}$, however varying trajectories and distances had similar results. The results are shown in degrees error for comparability.

The PSO parameters $c_{1}$ and $c_{2}$ were set to 2 , and the weight $w$ decreased linearly from 0.9 to 0.4 . The number of particles varied for trilateration and TARDIS, which were set to $C=50$ and $C=7$ respectively. Maximum iterations were 1000 and $f_{c}=10^{-12}$, for $T=5$ and $T=200$ respectively. The initial average was set to $K=30$. An important thing to note is that trilateration tries to minimize 3 variables where TARDIS only computes 2 , and was thus able to run 10.9 times faster. In the MATLAB simulation the algorithms had a refresh rate of $43.5 \mathrm{~Hz}$ and $476 \mathrm{~Hz}$ respectively.

In the experiments the OptiTrack motion capture system, which uses infrared technology and a set of cameras to track markers with submillimeter accuracy was used to produce 

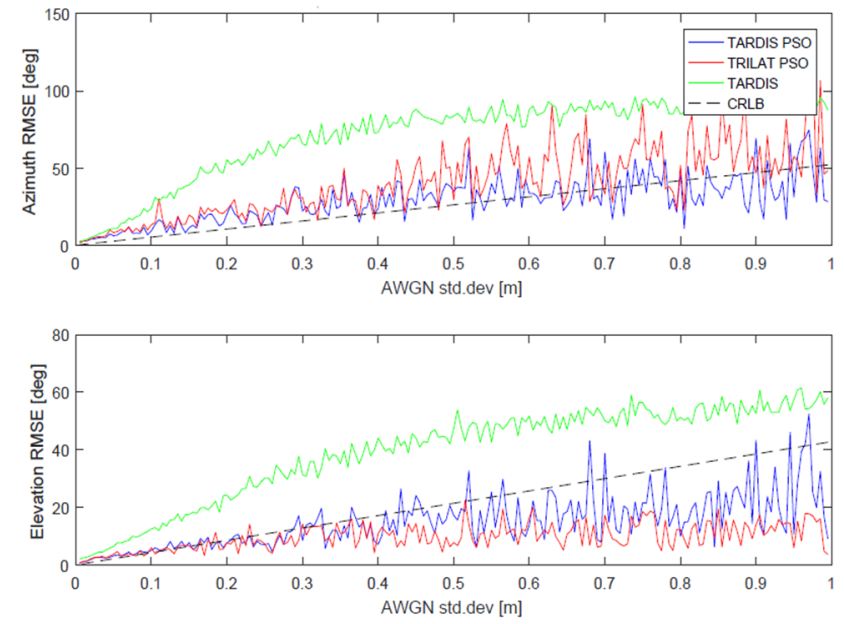

Figure 5. Directional RMSE of the algorithms with respect to the theoretical lower bound. Simulated for an arc trajectory over different levels of noise.

ground truth measurements. Due to time limitations only the TARDIS algorithm was implemented on the Mulle and the trilateration post-processed in MATLAB using the ranging results from the DWM1000 chips. Data was relayed via UART to the PC in this case, but does not influence the experiment itself. Although previously noted that the DWM1000 devices had ranging error with $\mathrm{AWGN}(0,0.05)$ for static cases, a big rotational bias was observed when moving them. This bias could be up to $\mu=0.5 \mathrm{~m}$ for different rotations of the antenna and thus had a significant impact on the positional estimation, due to the small baseline of the antenna array. An example of notable bias can be seen in Fig. 7, which has also been noted in other works that propose mitigation techniques [25], [26]. An overview of the positional estimation errors of an experiment can be seen in Fig. 6, and more details found in Table I.

All experiments had a somewhat different RMSE on the ranging input from the UWB devices, which is most likely due to different types of bias. Although conducted in a relatively clutter-free environment, ranging errors could still be present due to multipath reflections from the floor and partial NLoS
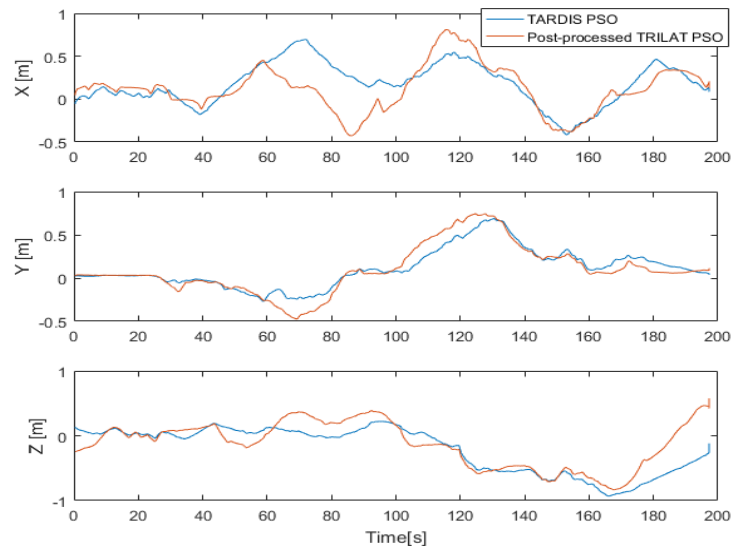

Figure 6. Positional errors for a square trajectory in YZ plane.
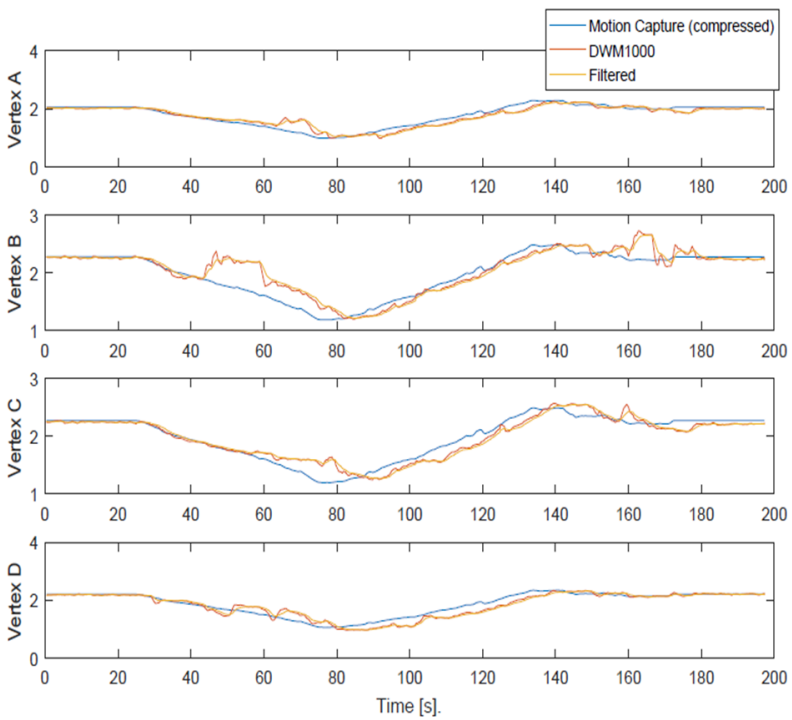

Figure 7. Range estimation from each vertex of the tetrahedron during an experiment, where bumps in range estimation are due to rotational bias.

from the structure itself. Furthermore [12] states that the ranging for these chipsets are dependent on the RSS. This is believed to be the cause for the rotational bias observed, due to the anisotropic radiation pattern of the antennae.

Positional accuracy achieved practically for both algorithms are thus around $50 \mathrm{~cm}$, which could be sufficient for certain types of swarm configurations. Although more precise approaches exist for relative localization the proposed technology is very low cost, omnidirectional and fully distributed, making it a suitable candidate for moderately sized swarm agents or other types of platforms.

The novel TARDIS algorithm currently has an update rate of about $10 \mathrm{~Hz}$ running on the Mulle. Thus, it is a better candidate than the more traditional trilateration algorithm that would run about 11 times slower according to simulations, which was also found in [17]. seriously restricting the relative velocity of the source node.

\section{CONCLUSION AND FUTURE WORK}

In this paper, we compare a novel technique for estimating the relative localization between moving nodes with a more traditional one. The simulation results show a similar accuracy for both algorithms, which attains and sometimes exceeds the CRLB for various degrees of AWGN. In the example implementation, a ranging bias is observed due to relative antenna orientation. This has a large impact on the positional estimation due to the small baseline of the sensor, yielding a RMSE of approximately $0.5 \mathrm{~m}$. The update frequency with TARDIS is observed to be 10.9 times faster than the more traditional trilateration algorithm, which will allow the relative velocity between nodes to be less restricted. Compared to visual based systems the proposed technology has lower resolution in terms of accuracy and refresh rate, but compensate for it by having a much greater range and environmental flexibility. Also 
as the system is very light weight and independent of GI, the developed localization sensor is a good alternative for e.g. moderately sized swarms or in an IoT setting, which can allow for a margin of error.

TABLE I. EXPERIMENTAL RESULTS WITH THE OPTITRACK MOTION CAPTURE SYSTEM USED AS GROUND TRUTH

\begin{tabular}{|l|c|c|c|c|c|c|}
\hline $\begin{array}{c}\text { Experimental } \\
\text { results }\end{array}$ & \multicolumn{2}{|c|}{ RMSE TARDIS (m) } & \multicolumn{3}{c|}{ RMSE TRILAT (m) } \\
\hline \multicolumn{1}{|c|}{ Trajectory } & $\boldsymbol{X}$ & $\boldsymbol{Y}$ & $\boldsymbol{Z}$ & $\boldsymbol{X}$ & $\boldsymbol{Y}$ & $\boldsymbol{Z}$ \\
\hline Square XY & 0.48 & 0.14 & 0.36 & 0.53 & 0.22 & 0.50 \\
\hline Square YZ & 0.30 & 0.24 & 0.40 & 0.40 & 0.36 & 0.47 \\
\hline Square XY & 0.63 & 0.47 & 0.77 & 0.63 & 0.51 & 0.76 \\
\hline Total & 0.47 & 0.28 & 0.51 & 0.52 & 0.36 & 0.58 \\
\hline
\end{tabular}

\section{ACKNOWLEDGMENT}

The authors would like to thank the Arrowhead and Productive 4.0 projects for funding.

\section{REFERENCES}

[1] G. P. Sadrollah, J.C Barca, A.I. Khan, J. Eliasson and I. Senthooran, "A distributed framework for supporting 3D swarming applications," Computer and Information Sciences (ICCOINS), International Conference on IEEE, 3-5 June 2014.

[2] L. Alboul, H. S. Abdul-Rahman, P. S. Haynes, J. Penders, and J. Tharin, "An Approach to multi-robot site exploration based on principles of self-organisation," Intelligent Robotics and Applications, vol. 6425. Springer, Berlin, Heidelberg, 2010.

[3] T. Stirling, J. Roberts, J. Zuerey, and D. Floreano, "Indoor navigation with a swarm of flying robots" Robotics and Automation (ICRA), 2012 IEEE International Conference on, pp. 4642-4647. IEEE, 14-18 May 2012.

[4] M, Saska, T. Baca, and J, Thomas et al, "System for deployment of groups of unmanned micro aerial vehicles in gps-denied environments using onboard visual relative localization," Auton Robot, 2016.

[5] C. McElroy, D. Neirynck, and M. McLaughlin, "Comparison of wireless clock synchronization algorithms for indoor location systems," Communications Workshops (ICC), 2014 IEEE International Conference on, pages 157-162. IEEE, 2014.

[6] S. Gezici and H. Vincent Poor, "Position estimation via ultrawide-band signals," Proceedings of the IEEE, 97(2):386-403, 2009.

[7] E. Staudinger, S. Zhang, A. Dammann, and C. Zhu, "Towards a radio-based swarm navigation system on mars key technologies and performance assessment," 2nd International IEEE Conference on Wireless for Space and Extreme Environments. IEEE, 3 Dec 2014.

[8] J. Valin, F. Michaud, and J. Rouat, "Robust 3d localization and tracking of sound sources using beamforming and particle altering," IEEE International Conference on Acoustics Speech and Signal Processing Proceedings, vol. 4, p. IV. IEEE, 2006.

[9] A. Maali, A. Ouldali, H. Mimoun, and G. Baudoin, "Uwb localization system with tdoa algorithm using experimental measurements," The Third International Conference on Advances in Satellite and Space Communications, pp. 97-102. IARIA, April 17-22 2011.
[10] S. Sand, W. Wang, and A. Dammann, "Cramr-rao lower bounds for hybrid distance estimation schemes," Proceedings of 76th IEEE Vehicular Technology Conference (VTC-Fall), Sep 2012.

[11] E. Karapistoli, F. Pavlidou, I. Gragopoulos, and I. Tsetsinas, "An overview of the ieee 802.15. 4a standard," IEEE Communications Magazine, 48(1):47-53, 2010.

[12] Decawave Ltd. DW 1000 user manual, version 2.09, 2016.

[13] V. Viswanathan, S. Jana, and S. Swarup, "Fast range-based localization of targets using particle swarm optimization," Control, Automation and Robotics (ICCAR), International Conference on, pp. 186-190. IEEE, 2015.

[14] P. H. Namin and M. A. Tinati, "Node localization using particle swarm optimization," Intelligent Sensors, Sensor Networks and Information Processing, Seventh International Conference on, pp. 288-293. IEEE,2011.

[15] Y. Zhou, "An efficient least-squares trilateration algorithm for mobile robot localization," Intelligent Robots and Systems, IEEE/RSJ International Conference on, pp. 3474-3479. IEEE, 2009.

[16] H. Guo, K. Low, and H. Nguyen, "Optimizing the localization of a wireless sensor network in real time based on a low-cost microcontroller," IEEE Transactions on Industrial Electronics, 58(3):741-749, 2011.

[17] Y. Qin, F. Wang, C. Zhou, and S. Yang., "A distributed particle swarm optimization based localization scheme in underground tunnels," Wireless Sensor Systems (WSS), IET Conference on, pp. 1-5. IET, 18 June 2012.

[18] S. Monica and G. Ferrari, "A swarm intelligence approach to $3 \mathrm{~d}$ distance-based indoor uwb localization," European Conference on the Applications of Evolutionary Computation, pp. 91-102. Springer, 2015.

[19] N.M. Kwok, D. K. Liu, and G. Dissanayake. "Evolutionary computing based mobile robot localization." Engineering Applications of Artificial Intelligence 19.8 (2006): 857-868.

[20] R. Janapati, Ch. Balaswamy and K. Soundararajan. "Enhanced mechanism for localization in wireless sensor networks using PSO assisted extended Kalman filter algorithm (PSO-EKF)." Communication, Information \& Computing Technology (ICCICT), 2015 International Conference on. IEEE, 2015.

[21] K. Acres and J.C. Barca, "Determining elevation and bearing information of a remote point," Pub.No WO/2017/063032 Int. App. No. PCT/AU2016/050949, 15 Oct 2015.

[22] S. G. Manyam, S. Rathinam, S. Darbha, D. Casbeer, Y. Cao, and P. Chandler, "Gps denied uav routing with communication constraints," Journal of Intelligent and Robotic Systems, 84(1-4), pp.691-703, 2016.

[23] R. Kyusakov, J. Eliasson, J. Delsing, J. van Deventer, and J. Gustafsson, "Integration of wireless sensor and actuator nodes with IT infrastructure using service-oriented architecture," IEEE Transactions on Industrial Informatics, 9(1), Feb 2013.

[24] Y. Shi. "Particle swarm optimization: developments, applications and resources," Evolutionary computation, Proceedings of the 2001 Congress on, vol. 1, pp. 81-86. IEEE, 2001.

[25] V. Barral, P. Surez-Casal, C. J. Escudero, and J. A. Garca-Naya, "Assessment of uwb ranging bias in multipath environments," International Conference on Indoor Positioning and Indoor Navigation (IPIN), Alcala de Henares, Spain, 2016.

[26] P. Vasilyev, S. Pearson, M. El-Gohary, M. Aboy, and J. McNames, "Inertial and time-of-arrival ranging sensor fusion," Gait \& posture, 54:1-7, 2017. 